\title{
Analysis of the main passive soft tissues associated with adult acquired flatfoot deformity development: A computational modeling approach
}

\author{
Christian Cifuentes-De la Portilla ${ }^{\mathrm{a}, \mathrm{c}}$, Ricardo Larrainzar-Garijo ${ }^{\mathrm{b}, *}$, Javier Bayod $^{\mathrm{a}}$ \\ ${ }^{a}$ Applied Mechanics and Bioengineering Group (AMB), Aragón Institute of Engineering Research (I3A), Universidad de Zaragoza, Spain \\ b Orthopaedics and Trauma Department, Medicine School, Universidad Complutense - Hospital Universitario Infanta Leonor, Madrid, Spain \\ ' Facultad de Ciencias Médicas, Departamento de Medicina, Universidad Espíritu Santo, Ecuador
}

\section{A R T I C L E I N F O}

\section{Article history:}

Accepted 29 December 2018

Available online $\mathrm{xxxx}$

\section{Keywords:}

Biomechanics

Flatfoot

FE modeling

Pes planus

\begin{abstract}
A B S T R A C T
Adult acquired flatfoot deformity (AAFD) is a pathology with a wide range of treatment options. Physicians decide the best treatment based on their experience, so the process is entirely subjective. A better understanding of soft tissue stress and its contribution in supporting the plantar arch could help to guide the clinical decision. Traditional experimental trials cannot consistently evaluate the contribution of each tissue. Therefore, in this research a 3-Dimensional FE foot model was reconstructed from a normal patient in order to measure the stress of the passive stabilizers of the arch, and its variation in different scenarios related with intermediate stages of AAFD development. All bones, the plantar fascia $(\mathrm{PF})$, cartilages, plantar ligaments and the spring ligament (SL) were included, respecting their anatomical distribution and biomechanical characteristics. An AAFD evaluation scenario was simulated. The relative contribution of each tissue was obtained comparing each result with a normal case. The results show that $\mathrm{PF}$ is the main tissue that prevents the arch elongation, while SL mainly reduces the foot pronation. Long and short plantar ligaments play a secondary role in this process. The stress increment on both PF and SL when one of two fails suggests that these tissues complement each other. These findings support the theory that regards the tibialis posterior tendon as a secondary actor in the arch maintenance, compared with the PF and the SL, because this tendon is overstretched by the hindfoot pronation around the talonavicular joint. This approach could help to improve the understanding of AAFD.
\end{abstract}

(c) 2019 Published by Elsevier Ltd.

\section{Introduction}

Adult Acquired Flatfoot Deformity (AAFD) has become a clinical research issue in recent years, motivated by the increasing prevalence of this pathology and the huge variability of currently available treatment alternatives (Lee et al., 2005; Shibuya et al., 2010). Depending on the stage of the pathology, doctors must decide between tendon reinforcement techniques, corrective osteotomies of the hindfoot valgus moment or selective arthrodesis in the middle and hindfoot joints (Fowble \& Sands, 2004; Guha \& Perera, 2012; Toullec, 2015). Therefore, currently there is no generally accepted consensus on how to act on patients in the intermediate stages (flexible arch deformity) (Bluman et al., 2007; Vulcano et al., 2013). The considerable variability in treatment is caused, in part, by a still latent lack of knowledge of certain biomechanical variables that have not been adequately evaluated by experimental trials, such as biomechanical tissue stress. The considerable

\footnotetext{
* Corresponding author.

E-mail address: rlarrainzar@gmail.com (R. Larrainzar-Garijo).
}

biomechanical complexity of the foot and the difficulty of segmenting and treating the tissue during experimental tests are the main causes of this problem (Huang et al., 1993; Rabbito et al., 2011).

Some researchers have described and evaluated normal or pathological human foot biomechanics using different kinds of foot models (Huang et al., 1993; Bertani et al., 1999; Cheung et al., 2005). Some cadaver experimental studies have been proposed, evaluating the reaction of the foot under progressive loads. These studies allow the measurement and evaluation of the anatomical deformation over both the foot bones and the soft tissues generated by the compression tests (Deschamps, et al., 2011; Toullec, 2015). Nevertheless, these approaches require high financial investments in measurement equipment, as well as a meticulous control over the study samples that guarantees the biomechanical characteristics of the tissue (Morales Orcajo et al., 2015).

One alternative accepted nowadays by clinicians and biomechanical engineers is Finite Element (FE) modeling. Technological advances have notably improved the processing capacity of computers, allowing complex numerical models to be designed for 
the study of human foot biomechanics (Wang et al., 2016a,b). These models are considered as a valid alternative since researchers can include variations and loads over virtual structures that cannot be easily considered when using real tissue (Smith, et al., 2016; Wang et al., 2016a,b; Smyth, et al., 2017; Wong, et al., 2017). Of course, their validity depends on the correct design of physiological structures and the realistic modeling of the mechanical tissue properties (Viceconti, et al., 2005). However, in most cases they do not consider certain tissues or tissue features that are required for analyzing AAFD development in depth (Wang et al., 2015).

Clinical studies have identified some passive stabilizers of the plantar arch with an important role in this pathology: the plantar fascia (PF) (Wang et al., 2016a,b), the short plantar ligament (SPL), the long plantar ligament (LPL) and the plantar calcaneonavicular ligament, also known as the spring ligament (SL) (Hidalgo et al., 2014; Wang et al., 2016a,b; Steginsky \& Vora, 2017). Currently published models simulate these tissues but without considering their geometry rigorously (Smith et al., 2016; Wang et al., 2016a,b). Most consider these tissues as bar elements (spring simple elements) that generate an adequate foot deformation but cannot measure relevant aspects such as the internal biomechanical stress generated in tissues under load and its distribution over the tissue geometries, reducing the clinical impact of these proposals in AAFD analysis (Smyth et al., 2017). This information is needed to know how much the tissue stress changes and what sections of the tissues are more loaded.

The objective of this research was to perform both a clinical and a biomechanical evaluation of AAFD development in the intermediate stages (IIa and IIb), characterized by a flexible arch deformity, using an innovative computational model of the foot. This allows an objective evaluation of the relative contribution of each passive stabilizer of the plantar arch. The results are comparable to those of experimental clinical tests. Additionally, this model is able to evaluate the subtalar divergence and both the fall and lengthening of the plantar arch (the main signs of AAFD), measured using the internal Moreau-Costa-Bertani angle (IMCB) and Kite's angle, which are common clinical metrics used in AAFD diagnostics.

\section{Methods}

This study is based on the model proposed by Morales Orcajo et al. (2015). The model reconstructs a normal human unloaded foot, based on tomography images (radiographs to $0.6 \mathrm{~mm} / \mathrm{slide}$ ) acquired from the right foot of a 49 year- old male, with a weight of $75 \mathrm{~kg}$ and a height of $170 \mathrm{~cm}$. The segmentation and tissue reconstruction were performed using MIMICS V. 10 software (Materialise, Leuven, Belgium).

\subsection{FE model design}

The model maintains the cartilage morphology and the plantar fascia as well as the differentiation of the cortical and trabecular bone, as shown in Fig. 1. This representation is generally overlooked by some authors, despite the importance of considering this differentiation in bone mechanics modeling, particularly in stress tissue evaluations (Garcia-Aznar et al., 2009; Bayod et al., 2012). For our model the SL, SPL and LPL were included based on anatomical images taken from atlases and cadaver dissection, under the advice of specialist foot and ankle surgeons. These tissues are fundamental for an adequate analysis of AAFD development.

\subsubsection{Meshing}

The model meshing was performed using the software ANSYS V.15 (Canonsburg, Pennsylvania, United States). A trial-error approach was employed to optimize the mesh size of each segment (Burkhart et al., 2013). The following conditions were considered in order to achieve a reasonable mesh size without compromising the calculation time: a minimum mesh size sufficiently small to fit into the tightest segments, a maximum mesh size consistent with the mínimum, to avoid large differences in element size between regions, a mesh accuracy of more than $99 \%$ of the elements being better than 0.2 mesh quality (Jacobians), and checking that the poor elements were located away from the region of greatest interest (PF, SL, LPL and SPL geometries) (See the bottom of Fig. 2).
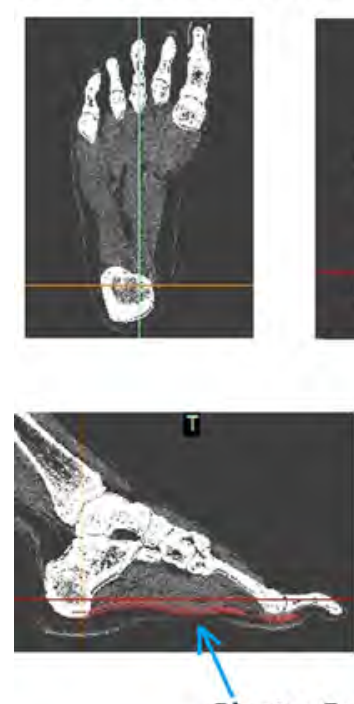

Plantar Fascia
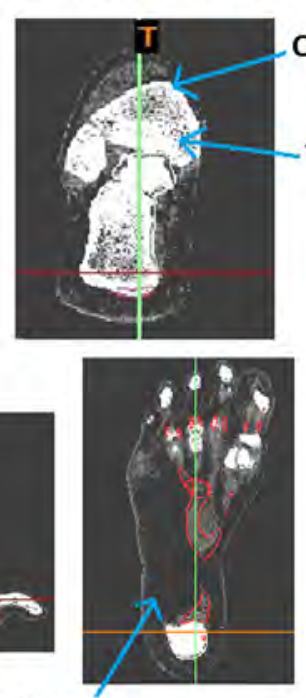

||IIIII)

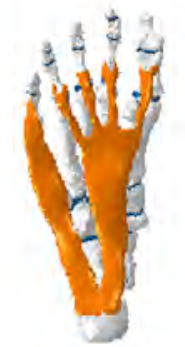

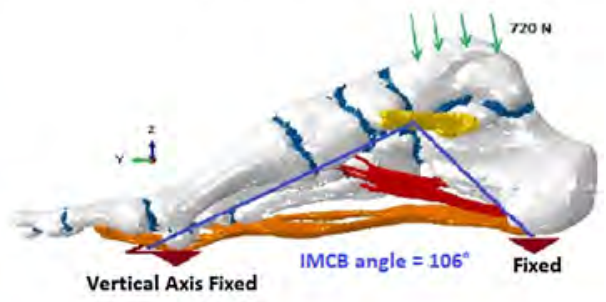

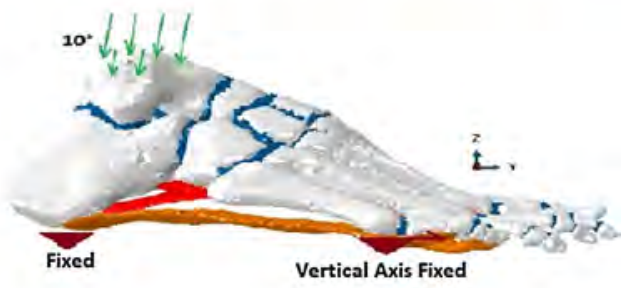

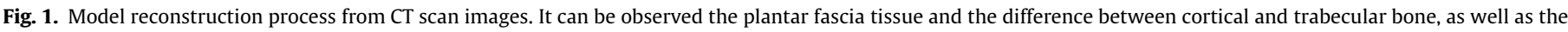
boundary and load conditions applied for simulations.

Please cite this article as: C. Cifuentes-De la Portilla, R. Larrainzar-Garijo and J. Bayod, Analysis of the main passive soft tissues associated with adult acquired flatfoot deformity development: A computational modeling approach, Journal of Biomechanics, https://doi.org/10.1016/j.jbiomech.2018.12.047 

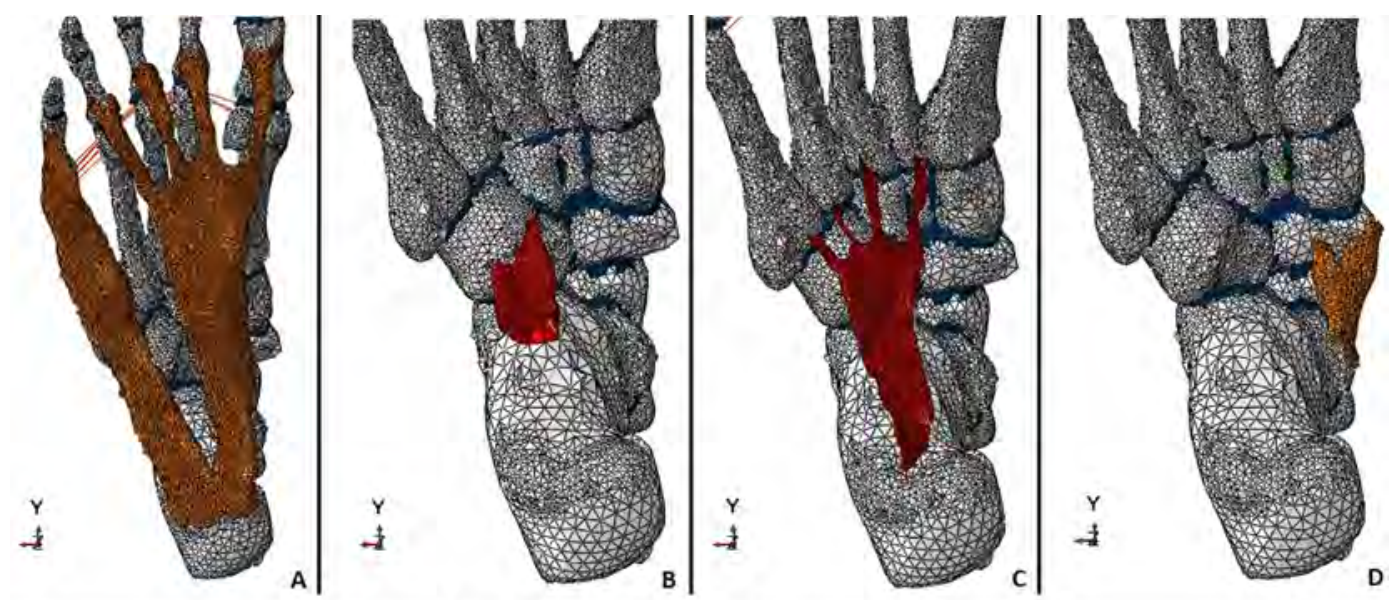

\section{Results for mesh quality evaluation Criteria: Min. angle $<30^{\circ}$}

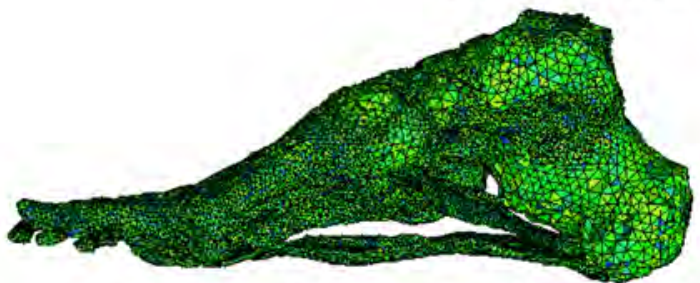

Accurate elements location $91.3 \%$

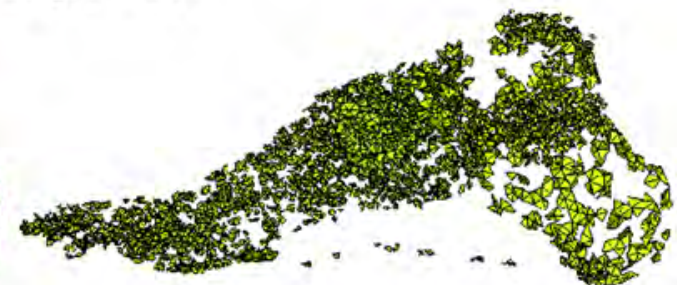

Inaccurate elements location

$8.7 \%$

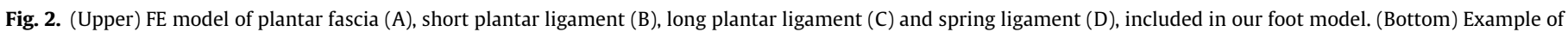
the elements location after applied one of the mesh quality evaluation criteria (Min angle <30 degrees).

The equilibrium was found with 247,453 linear tetrahedral elements (C3D4) with element sizes as follows: $1 \mathrm{~mm}$ for the smallest cartilages between phalanges, $2 \mathrm{~mm}$ for the phalanges, the thinnest ligaments and the rest of the cartilages, $3 \mathrm{~mm}$ for the metatarsals and the rest of the tendons, and $5 \mathrm{~mm}$ for the large bones in the hindfoot. All parameters were within good mesh quality ratios (see Table 1). The generated model is shown in Fig. 1. All the simulations were conducted with Abaqus 6.13 (Dassault Systémes, Vélizy-Villacoublay, France) using the Nonlinear geometry solver.

\subsection{Contour conditions}

The model reconstructs a non-weight foot (unloaded), thus an initial simulation to obtain a loading position was performed. The model was simulated including all the tissues using a $720 \mathrm{~N}$ load that represents the full weight of an adult person of about $73 \mathrm{Kg}$, leaning on one foot. This condition emulates a traditional AAFD diagnostic assessment scenario.

The load was introduced in a descending vertical direction, with 10 degrees of inclination (distributed in the zone of contact Tibia Astragalus (90\%) and Fibula - Astragalus (10\%)) (Morales Orcajo

Table 1

Mesh quality metrics based on Burkhart et al. (2013) recommendations.

\begin{tabular}{llll}
\hline Quality metric & $\begin{array}{l}\text { Assessment } \\
\text { criteria }\end{array}$ & $\begin{array}{l}\text { Accurate } \\
\text { elements }\end{array}$ & $\begin{array}{l}\text { Inaccurate } \\
\text { elements }\end{array}$ \\
\hline Element Jacobians & $>0.2$ & $99.7 \%$ & $0.3 \%$ \\
Aspect ratio & $<3$ & $93.5 \%$ & $6.5 \%$ \\
Min. angles & $>30^{\circ}$ & $91.3 \%$ & $8.7 \%$ \\
Max. angles & $>120^{\circ}$ & $98.8 \%$ & $1.2 \%$ \\
\hline
\end{tabular}

et al., 2015). The simulations were performed by maintaining fixed nodes at the lower part of the calcaneus and blocking the Z-axis displacement (vertical) of the lower nodes of the metatarsals (See Fig. 1). This was done in order to simulate the ground effect when an adult person is leaning on one foot.

\subsection{Tissue model}

The model includes the PF, LPL, SPL and SL in appropriate anatomical positions (Fig. 2).

These tissue models were considered such as an elastic-linear material, using biomechanical properties reported in the literature: Cortical bone $(E=17,000 \mathrm{MPa}, \quad v=0.3)$, trabecular bone $(E=700 \mathrm{MPa}, \quad v=0.3) \quad$ (Garcia-Aznar et al., 2009), ligaments $(E=250 \mathrm{MPa}, v=0.28)$ (Tao et al., 2010) and Plantar fascia ( $E=240 \mathrm{MPa}, v=0.28$ ) (Wright \& Rennels, 1964), $E$ being Young's modulus and $v$ Poisson's ratio. To model the cartilage tissue, a non-linear and hyperelastic model was used (Mansour, 2003; $\mathrm{Wu}, 2007)$. This was because some studies have demonstrated that this tissue displaces water under compression, thus it is not entirely correct to consider it as a quasi-incompressible material as is generally used in similar models. This dynamic behavior was represented using the Ogden model, following the suggestions reported by Mansour (2003) and Wu (2007). The Ogden model describes the hyperelastic behavior of rubber-like materials. Its strain energy density function $U$ is:

$U=\frac{\mu}{\alpha^{2}}\left(\lambda_{1}^{\alpha}+\lambda_{2}^{\alpha}+\lambda_{3}^{\alpha}-3\right)+\frac{1}{D}(J-1)^{2}$

where the initial shear modulus $\mu=4.4$, the strain hardening exponent $\alpha=2$ and the compressibility parameter $D=0.45$. 


\subsection{Evaluation criteria and simulation conditions}

Structural changes of the plantar arch were quantified calculating the IMCB angle and the talus-calcaneus divergence through the Kités angle. For this measurement, the application Ruler was used (Valencia, 2018).

To determine the plantar arch lengthening, the pronation of the hindfoot and the relative contribution of each tissue, we calculated the difference between each performed simulation and the results of the model in normal load conditions. To quantify the maximum deformation values of the model and obtain a relative comparison of each analyzed tissue, we performed a simulation eliminating all the foot tissues except the cartilaginous, following the methodology proposed by Tao et al. (2010) for a tissue experimental test using cadaver models. In this way, the maximum possible deformation of our model was obtained.

The foot fall was evaluated following the displacement of the lower part of the head of the talus, while the lengthening was calculated following the displacement of the lower point of the sesamoids.

Finally, in order to determine the biomechanical contribution of each tissue, the simulations were carried out maintaining and eliminating each one of the evaluated tissues. Although damaged tissues continue working after an injury, herein we wanted to identify how important each tissue is to maintain the foot arch in a normal position. The normal standing load was considered as a reference standard. Subsequently, the isolated effects on PF, SPL, LPL and SL were simulated, maintaining the same loading conditions.

\subsection{Foot FE model validation}

The model used for this study was validated following the recommendations of Tao et al. (2009), measuring some anatomical points under two different loading conditions (light loading and normal standing), from the lateral view. The variation of these points allows us to compare the vertical displacements visible in radiographic images of a normal foot with respect to the FE model predictions. We measured the foot lengthening (FL) and the vertical distance of the highest point of the Astragalous (AST) and the Navicular (NAV), the middle of the Cuneiform (CUN), and the highest point of the first metatarsal head (MTH1), as can be seen in Fig. 3. These measurements were performed using 12 radiographic images of 6 normal patients (light loading and full load test) to obtain a normalized average and standard deviation in order to perform an objective comparison with the model predictions.

\section{Results}

\subsection{Model validation}

The results of the validation process can be seen in Table 2 . The model generates a bone structure variation similar to that expected
Table 2

Results of the validation process. The values correspond to the difference between the measured distance from each point to the ground, under two different loading conditions: Light loading and normal standing load.

\begin{tabular}{llll}
\hline $\begin{array}{l}\text { Reference } \\
\text { point }\end{array}$ & $\begin{array}{l}\text { Model prediction } \\
(\mathrm{mm})\end{array}$ & $\begin{array}{l}\text { Patient average } \\
(\mathrm{mm})\end{array}$ & $\begin{array}{l}\text { Patient std. } \\
\text { deviation }\end{array}$ \\
\hline FL & 0.457 & 0.5 & 0.104 \\
AST & -0.292 & -0.291 & 0.03 \\
NAV & -0.33 & -0.278 & 0.056 \\
CUN & -0.324 & -0.205 & 0.122 \\
MTH1 & -0.056 & 0.094 & 0.014 \\
\hline
\end{tabular}

in a normal patient, considering the foot deformation from a lateral view under a light load (without soft tissue tension) and a normal standing load (soft tissue tension under normal conditions).

Additionally, the foot model deformation is within normal clinical ranges: the IMCB angle was between 115 and 125 degrees, and the Kite angle of divergence between 15 and 20 degrees (see Fig. 4 (A-B)). (C-D)

The maximum deformation of our model can be seen in Fig. 4

\subsection{Selective simulation of each evaluated tissue}

The IMCB and Kite's angles obtained from each selective simulation are summarized in Table 3.

The relative contributions of each tissue in both the fall and elongation of the plantar arch are shown in Table 4 . The values are presented in terms of deformation (in units of length) and relative difference (by percentage).

We measured the gradual deformation generated in each simulated case, as shown in Fig. 5. The deformation is represented in terms of the plantar arch decrease (right) and elongation (left) through a simulation step. Here the effect and contribution of each one of the tissues evaluated can be observed. We have called only bone the simulation performed by removing all the evaluated tissues, and Normal the normal loading simulation results. The similar effect observed in the simulations that evaluate both the SPL and LPL is remarkable.

\subsection{Plantar fascia and spring ligament stress variation}

Finally, we evaluate the stress changes over both the PF tissue and the SL (see Fig. 6). The evaluation was first performed evaluating the maximum principal stress keeping only the PF and SL separately and then removing each one of these tissues. The contribution of the LPC and LPL is relatively minor and was not considered. These material eigenvalues are closely related to the tensile stress that supports the tissues during loading. The scale was normalized using the normal standing load results in order to properly observe the differences in all of the cases evaluated: 71.60 KPa for PF and 61.1 KPa for SL.
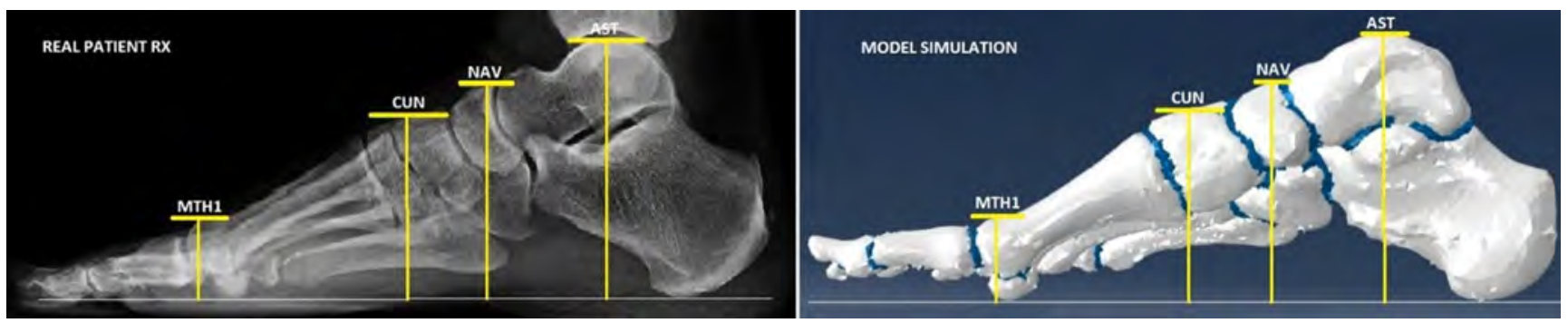

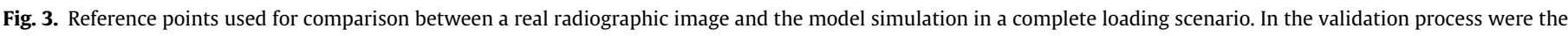
light loading scenario was also included.

Please cite this article as: C. Cifuentes-De la Portilla, R. Larrainzar-Garijo and J. Bayod, Analysis of the main passive soft tissues associated with adult acquired flatfoot deformity development: A computational modeling approach, Journal of Biomechanics, https://doi.org/10.1016/j.jbiomech.2018.12.047 


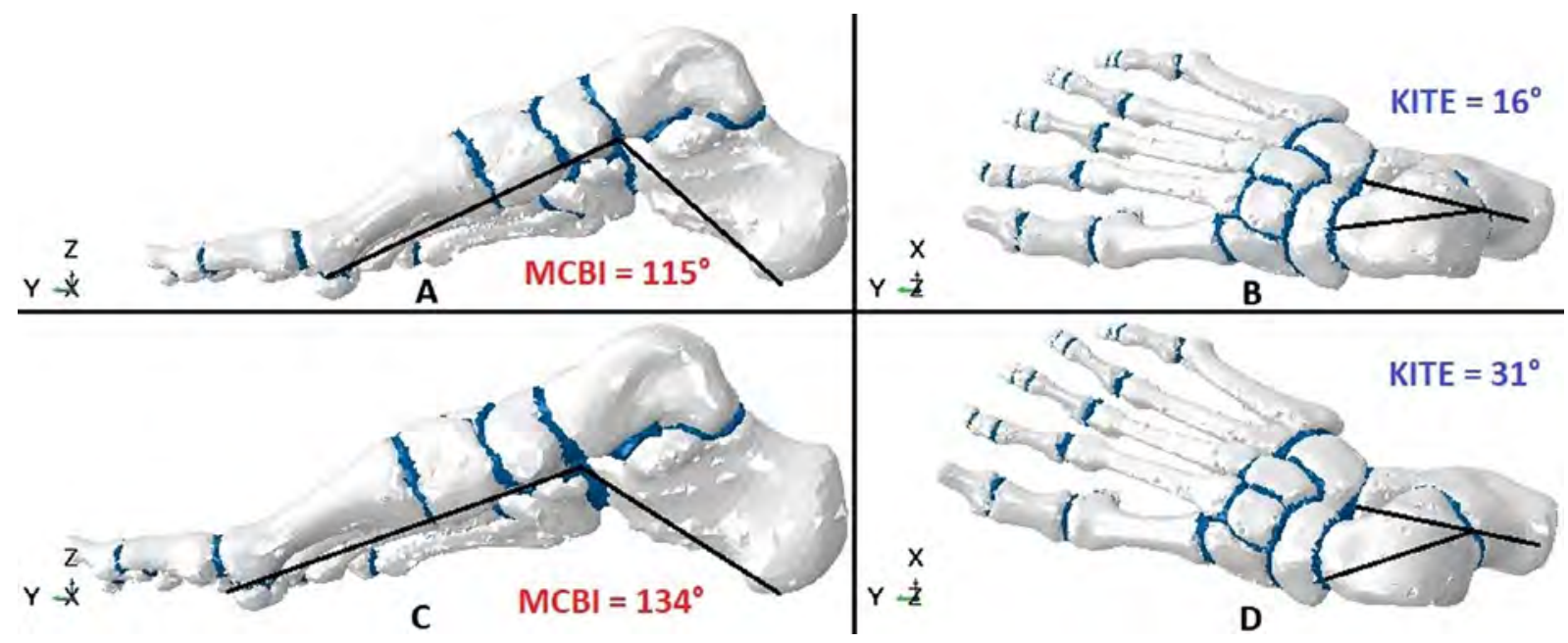

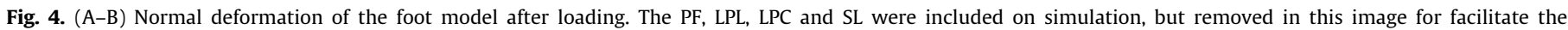
comparison. (C-D) Maximum deformation scenario obtained after remove all the passive soft tissues and maintaining only the bone structure and cartilages.

Table 3

Results summary. The normal standing load and Maximum deformation values were used as a reference to analyze the contribution of each tissue.

\begin{tabular}{lll}
\hline Test & IMCB angle $\left(^{\circ}\right)$ & Kite angle $\left(^{\circ}\right)$ \\
\hline Normal standing load & 115 & 16 \\
Maximum deformation & 134 & 31 \\
Removing PF & 121 & 18 \\
Keeping only PF & 119 & 20 \\
Removing SPL & 116 & 19 \\
Keeping only SPL & 125 & 22 \\
Removing LPL & 116 & 19 \\
Keeping only LPL & 124 & 24 \\
Removing SL & 117 & 24 \\
Keeping only LS & 122 & 17 \\
\hline
\end{tabular}

\section{Discussion}

This paper presents a computational human foot model that follows a clinical-oriented approach. This model is able to reproduce foot deformities in a loading position in both healthy and flatfoot cases. The soft tissues supporting the plantar arch were examined under different conditions. The versatility of the proposed model allowed us to evaluate the effect of removing the PF, SPL, LPL and SL and to assess their relative contributions in the plantar arch maintenance and the foot pronation, which are considered to be the main clinical signs associated with AAFD development.

This pathology affects the plantar arch, which provides support and deadening to the human gait (Gefen et al., 1999). In the clinical area, it is considered that the structures that maintain the plantar vault are the PF and the SL, and to a lesser extent the SPL and the LPL, but their contributions have not been clearly quantified. These results have already been reported by some researchers but based on experimental tests performed over real tissue (Huang et al., 1993; Tao et al., 2009). Our model is capable of obtaining results similar to those obtained in these studies but adding the quantification of the contribution of each tissue in the foot arch maintenance. Therefore, it can be used as a reference approach to studying diverse complex conditions that cannot be analyzed through experimental trials. Additionally, this model can be used in studies with more relevant clinical applications: the evaluation of surgical alternatives, specific designs of implants, individualized risk predictive tools, among others.

The normal standing load deformity obtained for the complete model simulation is both valid and adequate. The deformation results coincide with normal clinical values, evaluated in terms of the IMCB angle, Kite's angle and the bone structure deformation compared with radiographic measurements from a lateral view (Tao et al., 2009; Haddad et al., 2011).

Some authors have proposed computational models to evaluate AAFD and the effect of some tissues on the maintenance of the plantar arch. Gefen et al. (1999) and later Cheung and Zhang (2005) developed models to evaluate the biomechanical consequences of complete and partial removal of the PF. However, they considerably simplify the biomechanical characteristics of the tissues, as well as their geometry, assuming the PF as a purely deformable element (bar elements) (Cheung \& Zhang, 2005). Wu (2007) proposed a 2D model evaluating the longitudinal arch of the foot. This model considers a detailed anatomical structure, as well as a nonlinear model for cartilages. This work was the first FE approach to the evaluation of the plantar ligaments working on the maintenance of the plantar arch. Later, Tao et al. (2010) developed a detailed FE model which evaluated the FP, SL, LPC

Table 4

Results obtained from removing each evaluated structure (Upper) and maintaining only each evaluated tissue (Lower).

\begin{tabular}{|c|c|c|c|c|}
\hline \multirow[t]{2}{*}{ Model modification to perform each simulation } & \multicolumn{4}{|c|}{$\underline{\text { Relative difference }}$} \\
\hline & Fall (mm) & Elongation $(\mathrm{mm})$ & IMCB & Kite \\
\hline Removing PF & 0.27 (24.9\%) & $0.55(32.8 \%)$ & $6(31.6 \%)$ & $2(13.3 \%)$ \\
\hline Removing SPL & $0.003(0.3 \%)$ & $0.013(0.8 \%)$ & $1(5.3 \%)$ & $3(20 \%)$ \\
\hline Removing LPL & $0.024(2.2 \%)$ & $0.06(3.6 \%)$ & $1(5.3 \%)$ & $3(20 \%)$ \\
\hline Removing SL & $0.16(14.9 \%)$ & $0.36(21.4 \%)$ & $2(10.5 \%)$ & $6(53.3 \%)$ \\
\hline Maintaining only PF & $0.51(46.1 \%)$ & $0.86(51.5 \%)$ & $4(21.1 \%)$ & $4(26.7 \%)$ \\
\hline Maintaining only SPL & $0.77(69.7 \%)$ & $1.35(80.5 \%)$ & $10(52.6 \%)$ & $640 \%)$ \\
\hline Maintaining only LPL & $0.76(69.2 \%)$ & $1.28(76.6 \%)$ & $9(47.4 \%)$ & $8(53.3 \%)$ \\
\hline Maintaining only SL & $0.39(35.9 \%)$ & $0.83(49.9 \%)$ & $7(36.8 \%)$ & $1(6.7 \%)$ \\
\hline
\end{tabular}



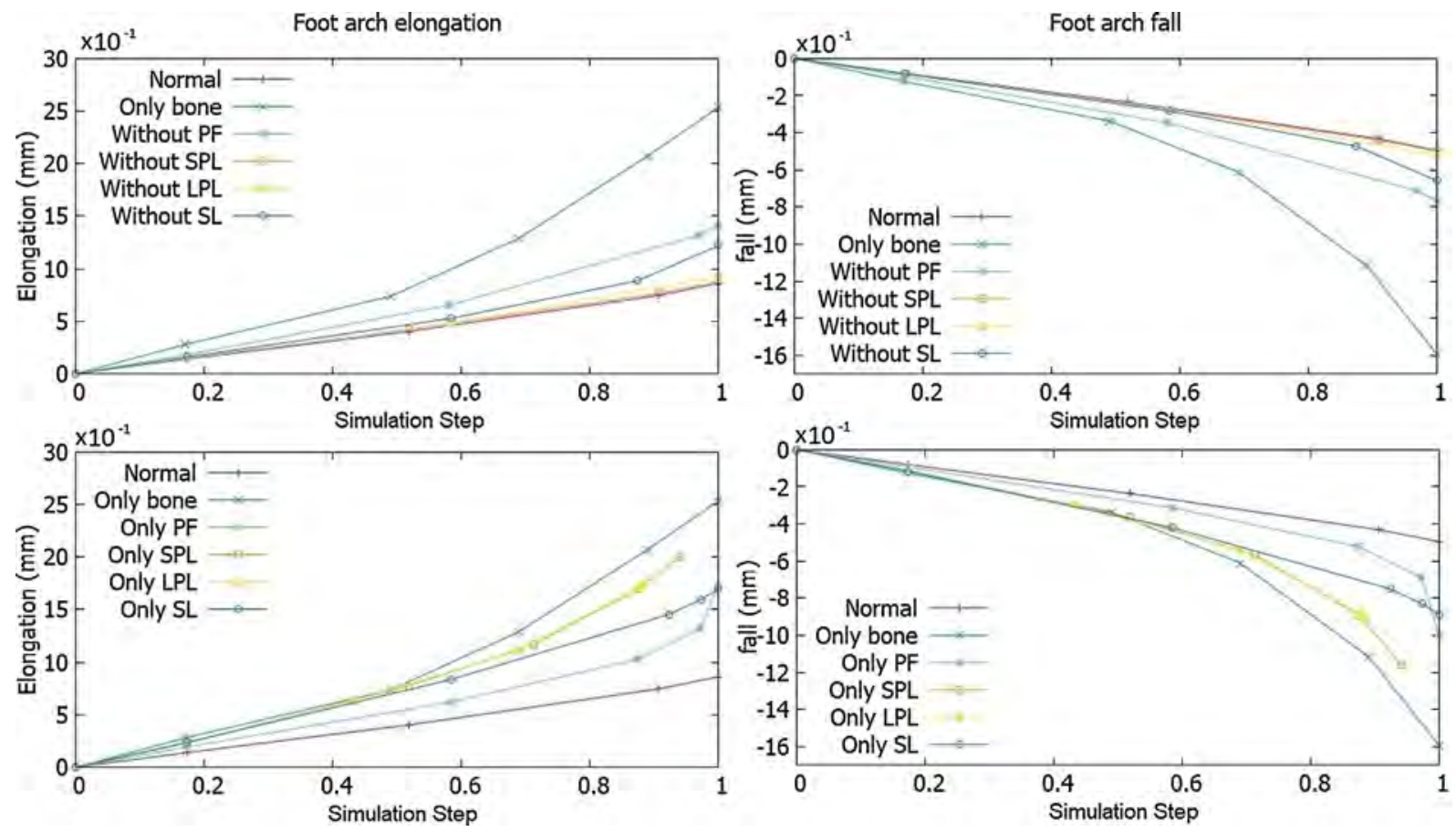

Fig. 5. Measurement of both the plantar arch elongation (right) and fall (left) obtained from simulations of each case evaluated in one simulation step.

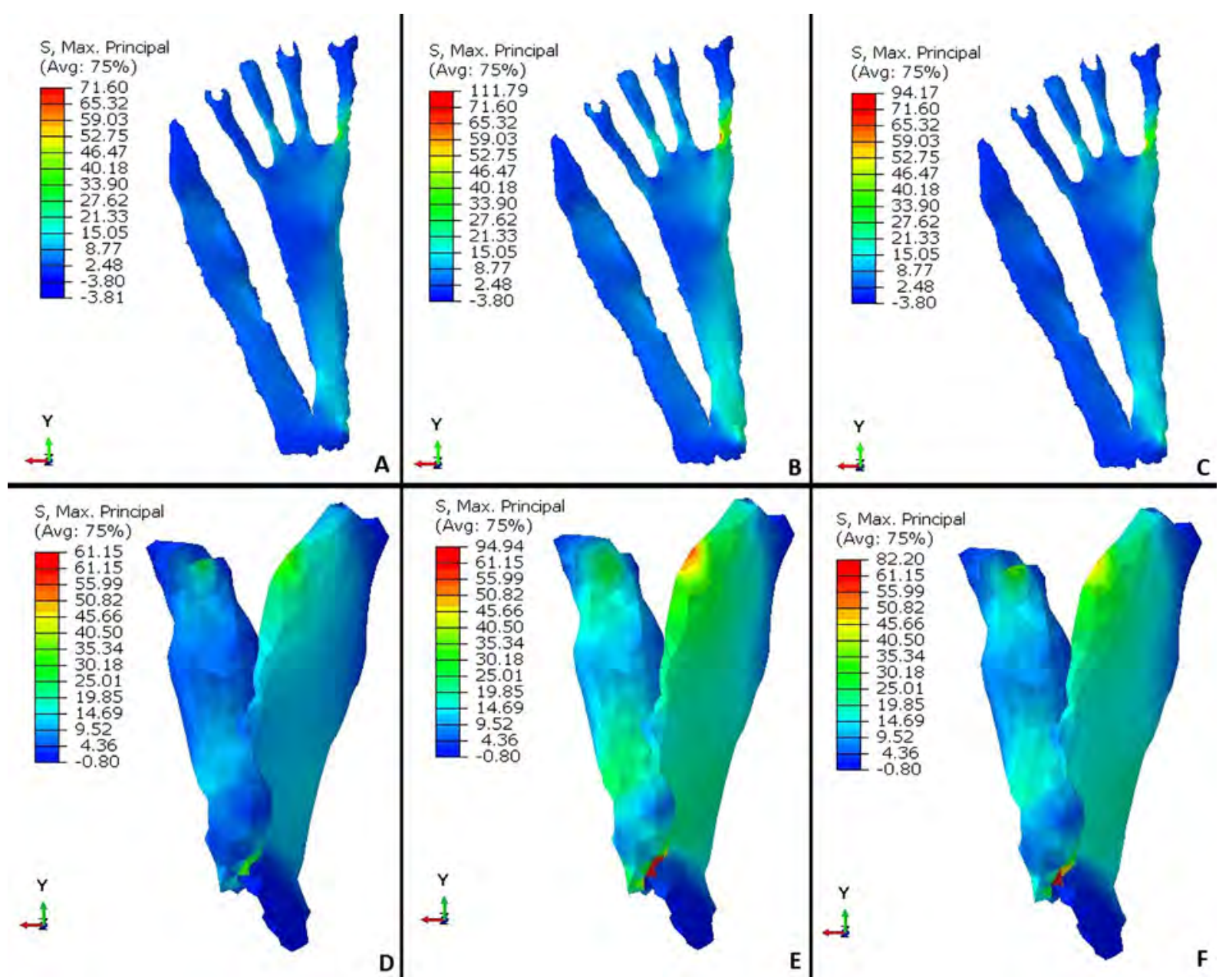

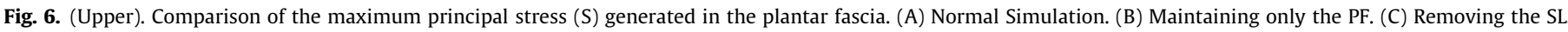
(Bottom) Comparison of the maximum principal stress (S) generated in the Spring Ligament. (D) Normal Simulation. (E) Maintaining only the SL. (F) Removing the PF.

Please cite this article as: C. Cifuentes-De la Portilla, R. Larrainzar-Garijo and J. Bayod, Analysis of the main passive soft tissues associated with adult acquired flatfoot deformity development: A computational modeling approach, Journal of Biomechanics, https://doi.org/10.1016/j.jbiomech.2018.12.047 
and LPL participation in the plantar arch maintenance. In contrast to our proposal, this author does not consider the hyperelastic behavior of the cartilage, the differentiation of the cortical and trabecular bone, nor the geometry suitable for the modeling of the evaluated tissues. The evaluation of Tao et al. (2010) was performed only in the absence of tissue and complements the assessment with an analysis of pressure differences on the metatarsals (Tao et al., 2010). Despite the aforementioned method differences, our results coincide in identifying the PF as the main tissue supporting the plantar arch, while the plantar ligaments play a secondary role. Additionally, our model can be used to quantify the tissue stress generated under different conditions, which is necessary in order to analyze and understand the effect of the pathologies associated with damage or weakening of the soft tissues in the foot. The results reported in Fig. 6 show that there is an important stress increase on the PF when the SL fails. Something similar happens in the results obtained from evaluating the SL stress. A notable increase in the tissue stress appears when the PF fails. These results suggest that both tissues play a fundamental role in the maintenance of the plantar arch. When one of the two fails, the other is noticeably overloaded.

Additionally, the results show that the PF is the tissue that contributes the most to prevent the falling of the plantar arch. These results are consistent with those reported by Huang et al. (1993) who made an evaluation of the contribution of each tissue experimentally on cadaveric feet. The similarity between this cadaver study and the results obtained in our simulations, show the validity of the mathematical prediction of the computational model approach. Additionally, our results showed that when a weakness or rupture of the PF occurs, there is a $24.9 \%$ increase in the arch fall (31\% CBI) but there is a variation of only 2 degrees (29\%) in the hindfoot pronation. Our results also show that the PF contribution is mainly in the reduction of the foot arch elongation, but it has little effect on the subtalar divergence.

On the other hand, it is noteworthy that our results show that injury or rupture of the SL could increase the plantar arch fall by about $14.9 \%$. This finding shows that the SL is the second most important tissue in the plantar maintenance. However, it has a more relevant effect, preventing forefoot pronation. In the simulations performed in the absence of SL, the model showed a variation of $53.3 \%$ in the Kités angle, which generates a pronounced foot pronation. Therefore, the fundamental contribution of this tissue is in the maintenance of the subtalar divergence rather than the lengthening of the foot.

The simulations also confirmed the secondary role of the LPL and SPL. A similar behavior was observed in the contribution in both cases. When simulating the model in the absence of these ligaments, there were no significant changes in either the fall or the elongation of the plantar arch. This was confirmed later in simulations performed maintaining only these tissues. It was observed that both the fall and pronation of the foot was considerable. Therefore, it can be concluded that these ligaments (due to their position and characteristics) cannot support the plantar arch on their own (Fig. 5). These results are similar to those obtained in experimental trials performed by Huang et al. (1993).

On the other hand, the model findings fit the theory that the tibialis posterior tendon has a secondary role compared to the PF and the SL in the plantar arch support. The clinical literature commonly suggests that tibialis posterior tendon insufficiency is the main factor causing AAFD. Some authors consider that the tibialis posterior tendon is overstretched by the hindfoot pronation around the talonavicular joint. This fact can be considered as the explanation for the association between tendon failure and clinical signs of adult flatfoot (Richie, 2007).

The main limitation of our model is that it considers the characterization of the PF, plantar ligaments and SL such as an elastic- linear material. However, since results are based on a static calculation with small displacements and deformations, the error is insignificant. Additionally, the model does not consider the plantar flat pad and plantar muscles of the foot because they do not have a known relationship with AAFD development or the passive plantar arch maintenance (Riddiford-Harland, et al., 2011). Experimental tests with similar objectives to our study performed using cadaver models do not report any effect of these tissues. Other muscles and their loads were not included, because our goal was to evaluate the passive stabilizers of the plantar arch.

Finally, we consider that the computational model presented herein could contribute to improve the biomechanical knowledge of human foot tissues and may be useful for future clinical research and analysis of AAFD development.

\section{Conclusion}

The proposed FE model can generate the foot deformation observed during AAFD evaluation. The results show that there is a significant increase in the stress over the PF when the SL fails and viceversa. These results suggest that both tissues play a fundamental role in the maintenance of the plantar arch. The PF is the main tissue that prevents foot elongation, while the SL mainly supports foot pronation. These findings are consistent with experimental studies which suggest that the tibialis posterior tendon is a secondary actor compared with the PF and the SL in AAFD development. This approach offers an option to clinical research for quantifying tissue strain, expanding the tools available for analyzing AAFD development.

\section{Acknowledgements}

The authors gratefully acknowledge the Spanish Ministry of Economy and Competitiveness for support through the project DPI2016-77016-R.

\section{Author disclosure statement}

The authors declare that they have no competing financial interests.

\section{Appendix A. Supplementary material}

Supplementary data to this article can be found online at https://doi.org/10.1016/j.jbiomech.2018.12.047.

\section{References}

Bayod, J. et al., 2012. Mechanical stress redistribution in the calcaneus after autologous bone harvesting. J. Biomech. 45, 1219-1226.

Bertani, A. et al., 1999. Flat foot functional evaluation using pattern recognition of ground reaction data. Clin. Biomech. 14, 484-493.

Bluman, E.M., Title, C.I., Myerson, M.S., 2007. Posterior tibial tendon rupture: a refined classification system. Foot Ankle Clin. 12 (2).

Burkhart, T.A., Andrews, D.M., Dunning, C.E., 2013. Finite element modeling mesh quality, energy balance and validation methods: A review with recommendations associated with the modeling of bone tissue. J. Biomech. 46, 1477-1488.

Cheung, J.T., Zhang, M., 2005. A 3-dimensional finite element model of the human foot and ankle for insole design. Arch. Phys. Med. Rehabil. 86, 353-358.

Cheung, J.T., Zhang, M., Leung, A.K.-L., Fan, Y.-B., 2005. Three-dimensional finite element analysis of the foot during standing-a material sensitivity study. J. Biomech. 38, 1045-1054.

Deschamps, K. et al., 2011. Body of evidence supporting the clinical use of 3D multisegment foot models: a systematic review. Gait Posture 33, 338-349.

Fowble, V.A., Sands, A.K., 2004. Treatment of adult acquired pes plano abductovalgus (flatfoot deformity): procedures that preserve complex hindfoot motion. Operat. Tech. Orthopaed. 14, 13-20.

Garcia-Aznar, J.M. et al., 2009. Load transfer mechanism for different metatarsal geometries: a finite element study. J. Biomech. Eng. 131, 021011. 
Gefen, A., Megido-Ravid, M., Itzchak, Y., Arcan, M., 1999. A 3-D numerical model of the human foot structure during gait, pp. 559.

Guha, A.R., Perera, A.M., 2012. Calcaneal osteotomy in the treatment of adult acquired flatfoot deformity. Foot Ankle Clin. 17, 247-258.

Haddad, S.L. et al., 2011. Adult acquired flatfoot deformity. Foot Ankle Int. 32, 95101.

Hidalgo, L.H. et al., 2014. Disfunción del tendón tibial posterior:? qué otras estructuras están implicadas en el desarrollo del pie plano adquirido del adulto? Radiología 56, 247-256.

Huang, C.K., Kitaoka, H.B., An, K.N., Chao, E.Y.S., 1993. Biomechanical evaluation of longitudinal arch stability. Foot Ankle 14, 353-357.

Lee, M.S. et al., 2005. Diagnosis and treatment of adult flatfoot. J. Foot Ankle Surg. $44,78-113$.

Mansour, J.M., 2003. Biomechanics of cartilage. Kinesiology: the mechanics and pathomechanics of human movement, pp. 66-79.

Morales Orcajo, E.. Barbosa de las Casas, E., Bayod López, J., 2015. Computational foot modeling for clinical assessment. Universidad de Zaragoza. PhD. Thesis.

Rabbito, M., Pohl, M.B., Humble, N., Ferber, R., 2011. Biomechanical and clinical factors related to stage I posterior tibial tendon dysfunction. J. Orthop. Sports Phys. Ther. 41, 776-784.

Richie, D.H., 2007. Biomechanics and clinical analysis of the adult acquired flatfoot. Clin. Podiatr. Med. Surg. 24, 617-644.

Riddiford-Harland, D., Steele, J., Baur, L., 2011. Medial midfoot fat pad thickness and plantar pressures: are these related in children? Int. J. Pediat. Obesity 6 (3), 261-266.

Shibuya, N. et al., 2010. Characteristics of adult flatfoot in the United States. J. Foot Ankle Surg. 49, 363-368.

Smith, B.A., Adelaar, R.S., Wayne, J.S., 2016. Patient specific computational models to optimize surgical correction for flatfoot deformity. J. Orthop. Res.
Smyth, N.A. et al., 2017. Adult-acquired flatfoot deformity. Eur. J. Orthop. Surg. Traumatol. 27, 433-439.

Steginsky, B., Vora, A., 2017. What to Do with the Spring Ligament. Foot Ankle Clin Tao, K. et al., 2010. Relative contributions of plantar fascia and ligaments on the arch static stability: a finite element study. Biomedizinische Technik/Biomed. Eng. $55,265-271$.

Tao, K. et al., 2009. An in vivo experimental validation of a computational model of human foot. J. Bionic Eng. 6, 387-397.

Toullec, E., 2015. Adult flatfoot. Orthopaed. Traumatol.: Surg. Res. 101, 11-17.

Valencia, U.P.d., 2018. Ergonautas. Available at: https://www.ergonautas.upv.es.

Viceconti, M., Olsen, S., Nolte, L.P., Burton, K., 2005. Extracting clinically relevant data from finite element simulations. Clin. Biomech. 20, 451-454.

Vulcano, E., Deland, J.T., Ellis, S.J., 2013. Approach and treatment of the adult acquired flatfoot deformity. Curr. Rev. Musculoskeletal Med. 6, 294-303.

Wang, Y., Wong, D.W.C., Zhang, M., 2016a. Computational models of the foot and ankle for pathomechanics and clinical applications: a review. Ann. Biomed. Eng. $44,213-221$.

Wang, Z. et al., 2015. FE Model of a Flatfoot Deformity for. Improving Surgical Planning.

Wang, Z., et al., 2016. Study of Surgical Simulation of Flatfoot Using a Finite Element Model. In: Innovation in Medicine and Healthcare 2015. s.l.: Springer, pp. 353363.

Wong, D.W.C. et al., 2017. Finite element simulation on posterior tibial tendinopathy: Load transfer alteration and implications to the onset of pes planus. Clin. Biomech.

Wright, D.G., Rennels, D.C., 1964. A study of the elastic properties of plantar Fascia. JBJS 46, 482-492.

$\mathrm{Wu}, \mathrm{L}$, , 2007. Nonlinear finite element analysis for musculoskeletal biomechanics of medial and lateral plantar longitudinal arch of Virtual Chinese Human after plantar ligamentous structure failures. Clin. Biomech. 22, 221-229. 